\title{
A New Strategy for Acid Anhydrides-modified Xylans in Ionic Liquids
}

\author{
Junli Ren ${ }^{1,2 *}$, Xinwen Peng ${ }^{1}$, Peng Feng ${ }^{3}$, and Runcang Sun ${ }^{3}$ \\ ${ }^{1}$ State Kay Laboratory of Pulp and Paper Engineering, South China University of Technology, Guangzhou 510640, China \\ ${ }^{2}$ Key Laboratory of Renewable Energy and Gas Hydrate, Chinese Academy of Sciences, Guangzhou 510640, China \\ ${ }^{3}$ Institute of Biomass Chemistry and Technology, Beijing Forestry University, Beijing 100083, China
}

(Received May 15, 2012; Revised July 1, 2012; Accepted July 1, 2012)

\begin{abstract}
Recently, homogeneous synthesis of functional carbohydrate polymers carried out in ionic liquids (ILs) has attracted extensive consideration because high reaction efficiency can be achieved. The objective of our work was to develop a new strategy to prepare acid anhydrides-modified xylan by the esterification of xylan with different types of acid anhydride such as acetic anhydride and succinic anhydride under the optimal dissolution condition of xylan in 1-butyl-3methylimidazolium chloride ([BMIM]Cl) ionic liquid. Significantly, effect factors on the xylan dissolution in [BMIM]Cl ionic liquid such as the concentration of xylan and the temperature were comparatively investigated, and the dissolution mechanism of xylan in [BMIM]Cl ionic liquid was revealed The physical and chemical properties of regenerated xylan were characterized by with various techniques such as FT-IR and GPC as well as TGA. Under the optimal dissolution condition of xylan in [BMIM] Cl, different acid anhydrides-modified xylan was comparatively discussed in the presence and the absence of catalyst. It was found that catalyst has different role in the chemical modification of xylan with different types of acid anhydride due to the chemical structure of acid anhydride.
\end{abstract}

Keywords: Xylan, Different types of acid anhydride, Ionic liquids, Dissolution, Catalyst

\section{Introduction}

Lignocellulose (mainly cellulose, hemicelluloses, and lignin) is a renewable raw material widely available in the form of waste from agricultural residues such as cereal straws and sugarcane bagasse, which present a large source of natural polymers [1]. Particularly, hemicelluloses are considered to be an almost inexhaustible source of raw material for the increasing demand of environmentally friendly and biocompatible products. Xylan is the main hemicelluloses component of the cell walls of hardwoods and herbaceous plants, and thus can be available in huge amounts as low cost products from forestry, agriculture, and pulp and paper industry. Xylan is amorphous, consists of branched chains of various sugars, and thus is easily dissolved in common solvents such as alkaline solution, dimethyl sulfoxide (DMSO), dimethylformamide/ lithium chloride (DMF/LiCl), which allows xylan being easily and homogeneously modified. Thus many increasing attempts have been made to develop xylan-based materials or biopolymers with functional groups in these solvents for potential application in industries [2-4].

Ionic liquids (ILs), as desirable green solvents and reaction media, have received significant attention. Homogeneous synthesis of functional carbohydrate polymers has been carried out in ILs, and high reaction efficiency can be achieved [5]. Ionic liquids mainly have been widely applied as solvent for cellulose [6-12]. In 2002, Swatloski et al first reported on 1-butyl-3-methylimidazolium chloride ([BMIM]Cl) as solvents for cellulose [6]. In 2003, a room-temperature ionic liquid RTIL, 1-allyl-3-methylimidazolium chloride
(AmimCl), was synthesized and proved to have outstanding capability for dissolving cellulose [7]. In 2005, another RTIL, 1(2-hydroxylethyl)-3-methylimidazolium chloride (HemimCl), was reported as a non-derivatizing solvent for cellulose [8]. More recently, it was reported that a series of alkylimidazolium salts containing dimethyl phosphate, methyl methylphosphonate, or methyl phosphonate could successfully dissolve cellulose at high concentrations and at room temperature [9]. In contrast to cellulose, the work to investigate ionic liquids to dissolve hemicelluloses has been comparatively limited [13-16]. The homogeneous chemical modifications of hemicelluloses such as the maleation [14], the acetylation [15] and succinication [16] were successfully carried out in [BMIM]Cl ionic liquid, indicating that ILs will be desirable alternatives to conventional solvents in preparation of novel biopolymers and biomaterials based on hemicelluloses.

In the present investigation, the aim is to develop was to develop a new strategy to prepare acid anhydride-modified xylan by the esterification of xylan with different types of acid anhydride under the optimal dissolution condition of xylan in [BMIM]Cl ionic liquid. The optimized dissolution condition of xylan in [BMIM]Cl ionic liquid was discussed by varying the dissolving temperature and the concentration of xylan in ionic liquid. The process of xylan dissolved in $\mathrm{BMIMCl}$ ionic liquid was monitored by polarizing microscope, and the characterization of regenerated xylan was characterized by Fourier transform infrared (FT-IR) and gel permeation chromatography (GPC) as well as thermogravimetric analysis (TGA). Furthermore, different resulting products were comparatively discussed in the presence and the absence of catalyst.

*Corresponding author: renjunli@scut.edu.cn 


\section{Experimental}

\section{Materials}

Xylan was isolated using $10 \% \mathrm{KOH}$ at $25^{\circ} \mathrm{C}$ for $10 \mathrm{~h}$ with a solid to liquid ratio of 1:20 $\left(\mathrm{g} \mathrm{m} l^{-1}\right)$ from holocellulose, which was obtained by the delignication of wheat straw with sodium chlorite in acidic solution ( $\mathrm{pH}$ 3.7-4.0, adjusted wtih $10 \%$ acetic acid) at $75^{\circ} \mathrm{C}$ for $2 \mathrm{~h}$. [BMIM]Cl ionic liquid was purchased from Shanghai Cheng Jie Chemical Co. Ltd. Acetic anhydride, succinic anhydride, succinic anhydride and other chemicals were purchased from Guangzhou Chemical Reagent Factory, China.

\section{The Dissolution of Xylan in Ionic Liquids}

The definite amount of xylan was added into the known amount of $[\mathrm{BMIM}] \mathrm{Cl}$ ionic liquid at required temperature during stirring. The process of dissolution for xylan in ionic liquid was detected by polarizing microscope.

\section{The Preparation of Regenerated Xylan from [BMIM]Cl Ionic Liquid}

The homogeneous mixture solution containing xylan was poured into the ethanol bulk (95\%) in a glass container and stirred for $30 \mathrm{~min}$ to form the precipitates. After filtration, the precipitates were washed by ethanol, centrifuged and dried at $45^{\circ} \mathrm{C}$ in a vacuum oven for $24 \mathrm{~h}$ to get the regenerated xylan.

\section{Different Acid Anhydrides-modified Xylan}

$0.33 \mathrm{~g}$ of dry xylan was added into $12.6 \mathrm{~g}$ of [BMIM]Cl ionic liquid, and then heated at $90{ }^{\circ} \mathrm{C}$ up to $1.5 \mathrm{~h}$ to guarantee the complete dissolution. Nitrogen gas was applied to prevent the entrance of $\mathrm{O}_{2}$. Then the required acid anhydride (acetic anhydride or succinic anhydride) and the definite amount of catalyst were added at the desirable temperature for the known reaction time. Finally, the mixture was cooled to room temperature, precipitated using $65 \%$ ethanol with stirring for $30 \mathrm{~min}$, and then centrifuged at $3500 \mathrm{rpm}$ for $20 \mathrm{~min}$. Then the precipitates were washed with $80 \%$ ethanol twice. The resulting products were dried at $45{ }^{\circ} \mathrm{C}$ in a vacuum oven for $24 \mathrm{~h}$. Note that If iodine as catalyst was added in [BMIM] $\mathrm{Cl}$ ionic liquid, after the reaction finished, the mixture was cooled to room temperature, and the solution of saturated sodium hyposulfite was added in the mixture, and then shaken until the black homogeneous mixture changed into a colorless flocculent appearance. Subsequently the mixture solution was precipitated, and the precipitates were washed and dried according to the method mentioned above.

\section{Determination of the Degree of Substitution (DS) of Modified Xylan \\ Determination of DS for Acetic Anhydride-modified Xylan}

The determination of DS for acetic anhydride-modified xylan was according to the method in our previous work [15]. The yield percentages were calculated based on the assumption that xylan was converted to diacetylated xylan. In the case the yield percentage would be $100 \%$. The DS for a xylan ester is defined as the moles of substituents of hydroxyl groups per D-xylopyranosyl structural unit of xylan, with two hydroxyl groups per unit. The theoretical maximum DS is 2.0. The unreacted acetic anhydride in a mixture of reaction was separated from the product by ethanol. If no reaction occurred and all of xylan was recovered unreacted, the yield percentage would be $60.9 \%$ with a DS value of 0.0 .

Determination of DS for Succinic Anhydride-modified Xylan

Two procedures were performed to determine the DS as follows [16]. (a) The purity of products. Product $(0.3 \mathrm{~g})$ was dissolved with $10 \mathrm{~m} l$ of water and stirred. Followed by adding hydrochloric acid $(1 \mathrm{M}, 10 \mathrm{~m} l)$, the mixture was agitated to dissolve completely. Five drops of phenolphthalein indicator were added into the mixture, and then sodium hydroxide $(1 \mathrm{M})$ was added dropwise with stirring until red color of the solution disappeared. Then, the mixture was poured into ethanol $(95 \%, 100 \mathrm{~m} l)$ under stirring and was left to settle for 15 min before the supernatant liquid was centrifuged. The precipitates were washed with $80 \%$ ethanol for four times and then washed again with ethanol $(95 \mathrm{wt} \%, 100 \mathrm{~m} l)$. Finally, the products were dialyzed (cutoff $=3500 \mathrm{~g} \cdot \mathrm{mol}^{-1}$ ) with distilled water and then freezedried. (b) Determination of DS of products. The average value of DS was determined by acidometric titration. Product $(0.05 \mathrm{~g})$ was dissolved in the distilled water $(50 \mathrm{~m} l)$ with a magnetic stirrer. The $\mathrm{pH}$ of the solution was adjusted to 8.00 by the addition of $\mathrm{NaOH}$ solution. Then the solution was titrated with $\mathrm{H}_{2} \mathrm{SO}_{4}(0.05 \mathrm{M})$ to $\mathrm{pH}$ 3.74. The DS was calculated based on the equations shown below.

$$
\begin{aligned}
& a=\frac{m^{\prime}}{m} \\
& B=\frac{2 M V}{a m} \\
& D S=\frac{132 B}{1-100 B}
\end{aligned}
$$

Where $a$ is the purity of the resulting product. $m$ and $m^{\prime}(\mathrm{g})$ are weights of product after and before puried, $132\left(\mathrm{~g} \cdot \mathrm{mol}^{-1}\right)$ is the molar mass of a xylose unit, $100\left(\mathrm{~g} \cdot \mathrm{mol}^{-1}\right)$ is the net increase in the mass of a xylose unit for each succinic anhydride substituted, $M=$ molarity of $\mathrm{H}_{2} \mathrm{SO}_{4}$ used, $V=L$ of $\mathrm{H}_{2} \mathrm{SO}_{4}$ used to titrate sample, $B=\mathrm{mol} \cdot \mathrm{g}^{-1}$ of $\mathrm{H}_{2} \mathrm{SO}_{4}$ consumed per gram of the products. All the titrations were carried out in triplicates and standard deviations were less than $4.0 \%$.

Characterization of Xylan and Regenerated Xylan

Xylan and regenerated xylan were determined by GPC on a PL aquagel-OH 50 column $(300 \times 7.7 \mathrm{~mm}$, Polymer 
Laboratories Ltd.), calibrated with PL pullulan polysaccharide standard (average peak molecular weights of 783, 12200, 100000,1600000 ). A flow rate of $0.5 \mathrm{ml} / \mathrm{min}$ was maintained. The eluent was $0.02 \mathrm{~N} \mathrm{NaCl}$ in $0.005 \mathrm{M}$ sodium phosphate buffer ( $\mathrm{pH}$ 7.50). Detection was achieved with a Knauer differential refractometer. The column oven was kept at $30{ }^{\circ} \mathrm{C}$. Xylan and regenerated xylan were dissolved with $0.2 \mathrm{M} \mathrm{NaCl}$ in $0.005 \mathrm{M}$ sodium phosphate buffer, $\mathrm{pH} 7.50$, both at the concentration of $0.1 \%$.

FT-IR transmission spectra of xylan and regenerated xylan were measured by using a Nicolet 750 spectrophotometer (Thermo Fisher Nicolet, Florida, USA) within the wave number range of $400-4000 \mathrm{~cm}^{-1}$, and the $1 \%$ finely ground samples were mixed with $\mathrm{KBr}$ to press a plate for measurement.

Thermal analysis of xylan and regenerated xylan was performed using TG/DTA analyzer (Pyris Diamond TG/DTA, PE Instrument). The apparatus was continually flushed with nitrogen. The sample weighed between 9 and $11 \mathrm{mg}$ and heated from room temperature to $600{ }^{\circ} \mathrm{C}$ at a heating rate of $10{ }^{\circ} \mathrm{C}$ per minute.

\section{Results and Discussion}

\section{The Effect of the Temperature on the Molecular Weight of Regenerated Xylan}

As well known, the parameters factors such as temperature and the concentration of xylan have significant influence on the dissolution process of xylan in ionic liquid, and thus affect the $M_{\mathrm{w}}$ of regenerated xylan. The effect of dissolving temperature on the molecular weight of regenerated xylan is shown in Table 1 . The melting point of [BMIM]Cl ionic liquid is $75^{\circ} \mathrm{C}$. When ionic liquids are used as solvents, the dissolving temperature should be above at least $10{ }^{\circ} \mathrm{C}$ of the melting point. So the dissolving temperature range of $90-110^{\circ} \mathrm{C}$ was studied. Obviously, temperature had great influence on the molecular weight of xylan. Compared with the isolated xylan with the $M_{\mathrm{w}}$ of $26800 \mathrm{~g} \cdot \mathrm{mol}^{-1}$ with a polydispersity of 2.93 , the $M_{\mathrm{w}}$ of regenerated xylan decreased from 24372 to $21838 \mathrm{~g} \cdot \mathrm{mol}^{-1}$ with an increment of temperature from 90 to $110{ }^{\circ} \mathrm{C}$. It meant that xylan occurred to be degraded to a certain extent during the dissolution process. With an increment in temperature, ionic liquid have great ability to dissolve xylan and simultaneously to degrade xylan, resulting in the decrease of $M_{\mathrm{w}}$ of the regenerated xylan at high temperature. Therefore, the dissolving temperature of $90{ }^{\circ} \mathrm{C}$

Table 1. Effect of temperature on the molecular weight of regenerated xylan

\begin{tabular}{cccc}
\hline Temperature $\left({ }^{\circ} \mathrm{C}\right)$ & $M_{\mathrm{w}}$ & $M_{\mathrm{n}}$ & $M_{\mathrm{w}} / M_{\mathrm{n}}$ \\
\hline 90 & 24372 & 5442 & 4.47 \\
95 & 22887 & 9435 & 2.42 \\
100 & 22135 & 9057 & 2.44 \\
110 & 21838 & 8743 & 2.62 \\
\hline
\end{tabular}

Table 2. Effect of xylan concentration on the molecular weight of regenerated xylan

\begin{tabular}{cccc}
\hline Concentration (\%) & $M_{\mathrm{w}}$ & $M_{\mathrm{n}}$ & $M_{\mathrm{w}} / M_{\mathrm{n}}$ \\
\hline 1.0 & 23981 & 13417 & 1.78 \\
2.6 & 24372 & 5442 & 4.47 \\
5.0 & 22379 & 9739 & 2.40 \\
7.5 & 21267 & 8895 & 2.50 \\
10.0 & 19131 & 6085 & 3.31 \\
\hline
\end{tabular}

was moderate considering the $M_{\mathrm{w}}$ of regenerated xylan.

\section{The Effect of Xylan Concentration on the Molecular Weight of Regenerated Xylan}

Table 2 shows the effect of the xylan concentration in ionic liquid on the molecular weight of regenerated xylan. The dissolving temperature was fixed at $90{ }^{\circ} \mathrm{C}$, and the dissolving time was $1.5 \mathrm{~h}$. The molecular weights of regenerated xylan were lower than that of native xylan, indicating that during the dissolution process xylan occurred to be degraded to a different extent. Increasing the concentration of xylan, the $M_{\mathrm{w}}$ of regenerated xylan increased and then decreased. In the process of dissolution, dissolution and degradation occurred simultaneously. At low concentration, the dissolution of xylan rapidly ran, and the degradation might be preponderant over the dissolution with prolonging the time. However, at higher concentration, the $M_{\mathrm{w}}$ of regenerated xylan contrarily decreased. Maybe [BMIM]Cl ionic liquid had higher ability to dissolve xylan and to degrade xylan in the limited time. In addition, too much amount of xylan was added in ionic liquid, the viscosity of medium increased. Therefore, the $2.6 \%$ concentration of xylan in ionic liquid was appropriate in view of the cost and $M_{\mathrm{w}}$ of regenerated xylan.

\section{Mechanism of Ionic Liquid to Dissolve Xylan}

Figure 1 shows the pictures taken at the different time in the dissolution process of xylan in ionic liquid by polarizing microscope. There were more bright points in Figure 1(a), which meant that the crystal region of xylan was reflected and consequently caused them. The image in Figure 1(b) was taken after $70 \mathrm{~min}$. Apparently, there were less bright points than that in picture a. the crystal region was destroyed by ionic liquid, and most of $\mathrm{x} / \mathrm{ylan}$ was dissolved. The image in Figure 1(c) was taken after 90 min. the black filed was present, indicating that xylan was dissolved completely. Thus ionic liquid has the great ability to completely dissolve xylan to form the homogenous system at shorter time, in comparison with cellulose dissolution in ionic liquid.

In view of the dissolution mechanism of cellulose in ILs [17], the proposed dissolving mechanism of xylan in ILs is brought forward (Figure 2). The oxygen and hydrogen atoms of $\mathrm{OH}$ in the xylan chain serve as electron donors and electron acceptors to form electron donor-acceptor (EDA) complexes with the cations and anions of ILs, respectively. 

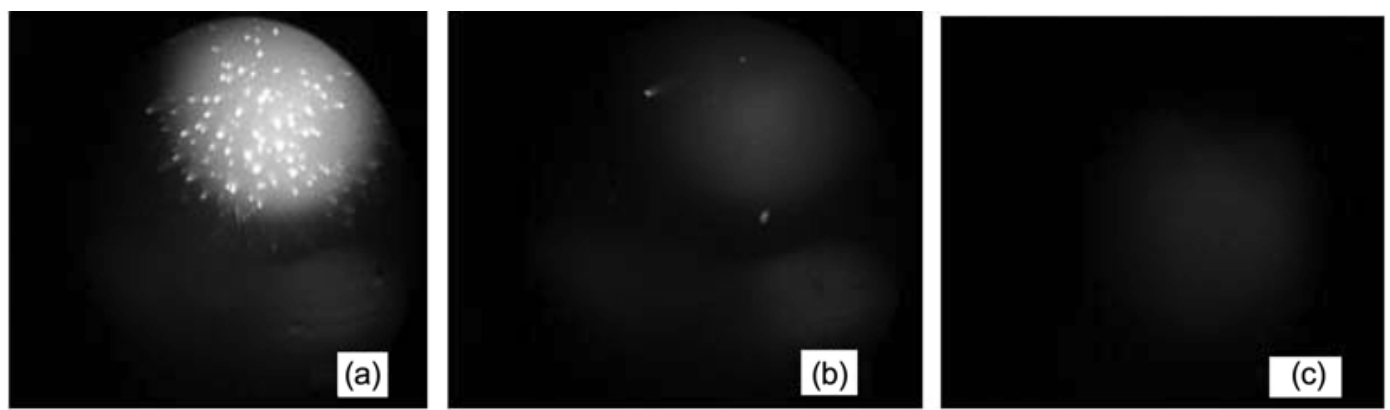

Figure 1. The pictures of xylan dissolution in ionic liquids taken by polarizing microscope (a for $0 \mathrm{~min}, \mathrm{~b}$ for $70 \mathrm{~min}$, and $\mathrm{c}$ for $90 \mathrm{~min}$ ).

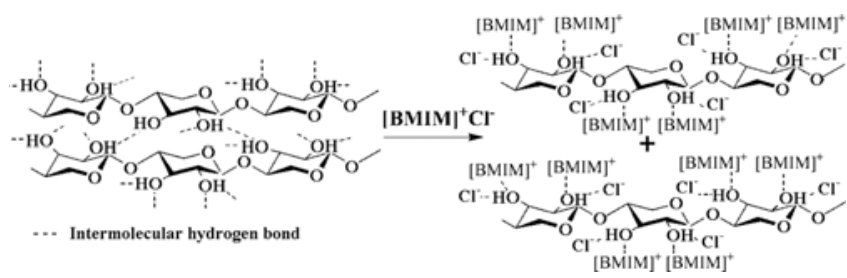

Figure 2. Proposed dissolution mechanism of xylan in [BMIM]Cl ionic liquid.

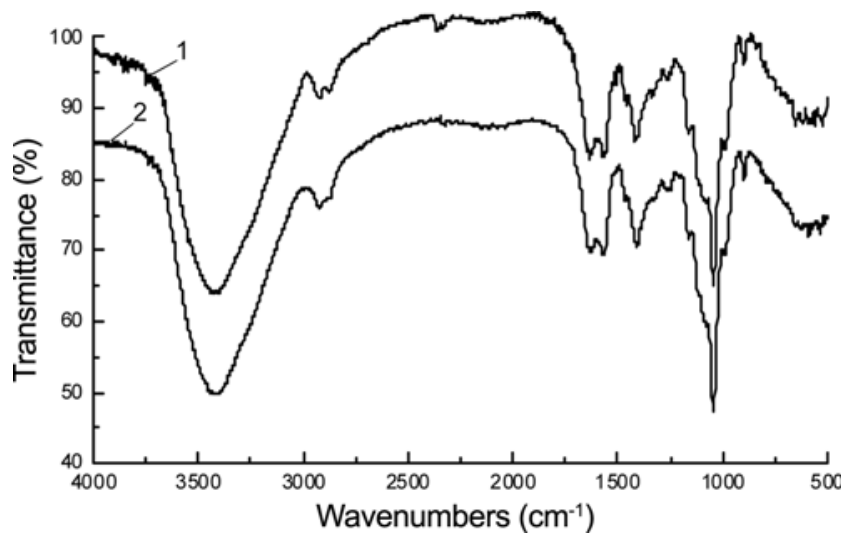

Figure 3. FT-IR spectra of regenerated xylan (spectrum 1) and native xylan (spectrum 2).

This interaction efficiently destroyed the hydrogen bonds among different xylan chains, resulting in the dissolution of xylan in ionic liquids.

\section{FT-IR Spectra of Xylan and Regenerated Xylan}

To detect the derivatation occurred on xylan in the dissolution process, FT-IR spectra of native and regenerated xylan are shown in Figure 3. It can be seen that the profile of two spectra was similar, which meant that [BMIM]Cl ionic liquid had the function to dissolve xylan as a nonderivatizing solvent.

\section{Thermal Analysis}

The thermostability of xylan and regenerated xylan is shown in Figure 4. As can be seen from the TGA plot of

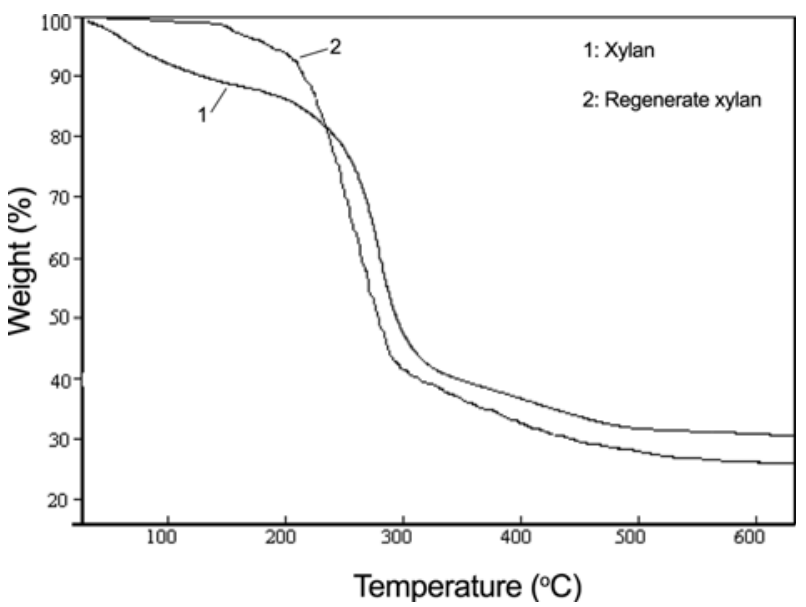

Figure 4. The thermostability of xylan and regenerated xylan.

xylan (curve 1) and regenerated xylan (curve 2), for all samples studied, the first weight loss occurred between 50 and $200{ }^{\circ} \mathrm{C}$, which are attributed to the evaporation of water. As $10 \%$ weight losses, the decomposition temperatures were $132^{\circ} \mathrm{C}$ and $218^{\circ} \mathrm{C}$ for xylan and regenerated xylan, respectively. As $40 \%$ weight losses, the decomposition temperatures of xylan and regenerated xylan occurred at $282^{\circ} \mathrm{C}$ and $273{ }^{\circ} \mathrm{C}$. As $50 \%$ weight losses, the decomposition temperatures were $294{ }^{\circ} \mathrm{C}$ and $283^{\circ} \mathrm{C}$ for xylan and regenerated xylan, respectively. At the range from $232{ }^{\circ} \mathrm{C}$ to $600{ }^{\circ} \mathrm{C}$, the thermal stability of regenerated xylan was lower than that of xylan, which could be explained that ionic liquid dissolved xylan, the crystal region disappeared, and the inter-structure of xylan changed. At the temperature of $600{ }^{\circ} \mathrm{C}$, the residual weight of xylan was $31 \%$, while the residual weight of regenerated xylan was $15 \%$. In an inert atmosphere, the end-products of decomposition of xylan and regenerated xylan are carbonaceous residues [18]. The salts formed during the extraction and purification process will contribute to the ash [19]. From these results regenerated xylan had a little higher purity than isolated xylan. Therefore, it indicated that the thermostability of regenerated xylan from ionic liquid under the desirable dissolution condition decreased above $232{ }^{\circ} \mathrm{C}$, but increased below $232{ }^{\circ} \mathrm{C}$. These results 
contribute to preparing xylan film from ionic liquid with high thermostability.

\section{Comparatively Investigation of Different Anhydrides- modified Xylan in the Presence or the Absence of Catalyst}

Most of the organic and polymerization reactions are carried out in organic solvents of which some eventually end up polluting environment by evaporation or leakage. Ionic liquids, which are recyclable and environmentally compatible, may replace the conventional organic solvents. In this work, it was found that the desirable dissolution condition for xylan in [BMIM]Cl ionic liquid was the xylan concentration of $2.6 \mathrm{wt} \%$, temperature of $90{ }^{\circ} \mathrm{C}$ and time of $1.5 \mathrm{~h}$. Thus this optimal dissolution condition was applied in the esterification of xylan with different types of acid anhydride. The chemical reaction of xylan with acetic anhydride and succinic anhydride is illustrated in Figure 5. Although iodine is solid, it could be dissolved in [BMIM]Cl ionic liquid within several seconds at high temperature. Figure 6 shows the DS of different acid anhydrides-modified xylan under given condition in the presence or an absence of iodine as catalyst. For acetic anhydride-modified xylan, the reaction conditions given were at the 10 molar ratios of acetic anhydride to anhydroxylose unit in xylan, at the temperature of $100{ }^{\circ} \mathrm{C}$ and the run time for $30 \mathrm{~min}$. An increasing dosage of iodine

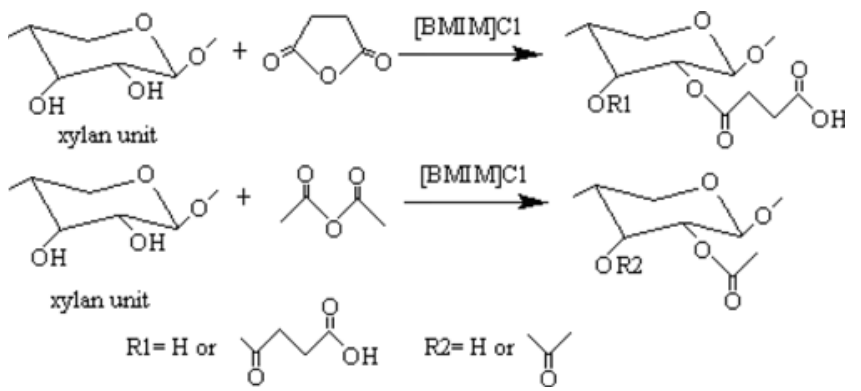

Figure 5. Scheme of chemical modification of xylan with acetic anhydride and succinic anhydride.

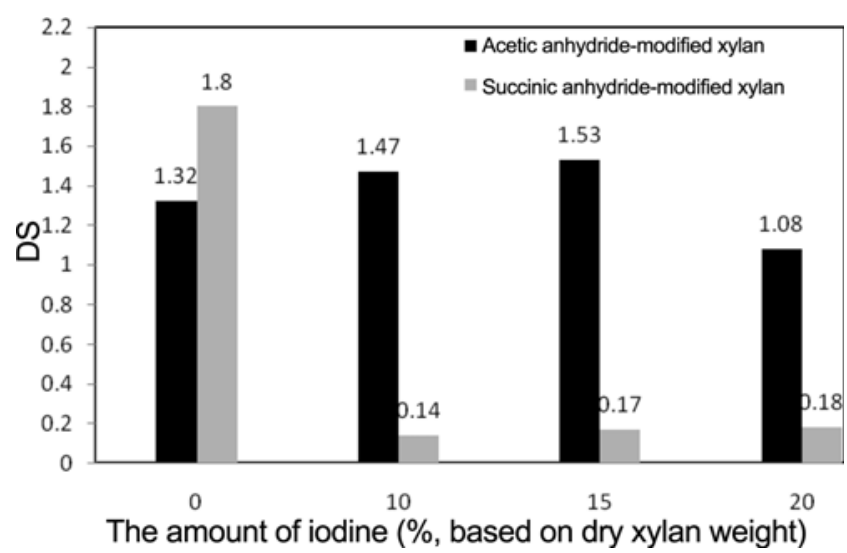

Figure 6. DS of acetic anhydride-modified xylan and succinic anhydride-modified xylan under given condition. from $0 \%, 10 \%$ and to $15 \%$ (based on dry xylan weight) led to a growth in DS. In contrast, further increasing the iodine dosage from $15 \%$ to $20 \%$ resulted in a decrement in DS, by 0.45 , respectively. Use of $15 \%$ iodine at the 10 molar ratios of acetic anhydride to anhydroxylose unit in xylan at $100{ }^{\circ} \mathrm{C}$ for $30 \mathrm{~min}$ led to increase in DS value by 0.21 , comparing to the DS of sample prepared without catalyst. These results showed that iodine substantially accelerated the rate of reaction for the esterfication of xylan with acetic anhydride. However, the contrary results occurred on succinic anhydridemodified xylan. Comparing to other samples obtained in the presence of iodine, higher DS (1.80) could be achieved under the absence of iodine at $100{ }^{\circ} \mathrm{C}$ for $40 \mathrm{~min}$ at the 6 molar ratios of succinic anhydride to anhydroxylose unit in xylan. The different role of iodine for the esterification of xylan with acetic anhydride or succinic anhydride might be due to the structure of acid anhydride, and it should be studied in a further detains.

To further study the influence of catalyst on the DS of succinic anhydride-modified xylan, catalysts such as $\mathrm{N}$ bromosuccinimide (NBS) and 4-dimethylaminopyridine (DMAP) were considered in this study, which is shown in Table 3. The other reaction parameters were unchanged except for the amount of NBS and DMAP. With an increment in the amount of catalysts from $6 \%$ to $15 \%$ and from $6 \%$ to $18 \%$ for NBS and DMAP, respectively, DS of succinic anhydride-modified xylan raised slightly. However, these DS values were much lower than that obtained without catalyst (1.80), indicating catalyst had negative impact on the chemical reaction of xylan with succinic anhydride in $[\mathrm{BMIM}] \mathrm{Cl}$ ionic liquid, and the reason was not clear but need to be further investigated in the next work. The succinic anhydride is extremely reactive, and will rapidly react with hydroxyl groups on xylan. So the higher DS could be achieved without the addition of catalyst. Another important reason was the excellent dissolving capacity and catalytic effect of ionic liquid. In the esterification of xylan in ionic liquids, the carbonyl groups are activated by bonding with ionic liquid [20], so the chemical reaction efficiency is significantly promoted without the addition of catalysts.

Table 3. The effect of different catalysts on DS of succinic anhydride-modified xylan

\begin{tabular}{ccc}
\hline Sample no. & Amount of catalyst $^{\mathrm{a}}$ & DS \\
\hline 1 & NBS 6\% & 0.44 \\
2 & NBS 10\% & 0.50 \\
3 & NBS 15\% & 0.46 \\
4 & DMAP 6\% & 0.56 \\
5 & DMAP 8\% & 0.59 \\
6 & DMAP 12\% & 0.64 \\
7 & DMAP 18\% & 0.61 \\
\hline
\end{tabular}

${ }^{a}$ Amount of catalyst was based on the dry xylan weight (\%). 


\section{Conclusion}

The development of the esterification reaction of xylan with different types of acid anhydride makes the use of $[\mathrm{BMIM}] \mathrm{Cl}$ as homogeneous system feasible. The present method had obvious advantages because the reaction was more rapid and complete. It was found that wheat straw xylan was completely dissolved in ionic liquid at $2.6 \%(\mathrm{w} / \mathrm{w})$ concentration at $90^{\circ} \mathrm{C}$ for $1.5 \mathrm{~h}$, and [BMIM] Cl ionic liquid had the function to dissolve xylan as a nonderivatizing solvent. In comparison to conventional solvents, higher DS for acetylated xylan (1.53) and succinoylated xylan (1.80) can be achieved at shorter time scale in [BMIM]Cl ionic liquid, which indicates that $[\mathrm{BMIM}] \mathrm{Cl}$ is an ideal reaction medium for the functionalization of xylan. Therefore, ionic liquids will create a completely homogenous system for the esterification of xylan, and accelerate the chemical reaction, resulting in rapid and efficient chemical functionalization of xylan.

\section{Acknowledgment}

This work was supported by the grants from Foundation for the Author of National Excellent Doctoral Dissertation of China (201169), National Natural Science Foundation of China (No. 31070530), Project of Guangzhou Science and Technology Plan (11C52080723/2011J4100065), Key Laboratory of Renewable Energy and Gas Hydrate, Chinese Academy of Sciences (No. y207ka) and the Fundamental Research Funds for the Central Universities (2012ZZ0081), SCUT.

\section{References}

1. J. Lundqvist, A. Teleman, L. Junel, G. Zacchi, O. Dahlman, F. Tjerneld, and H. Stalbrand, Carbohyd. Polym., 48, 29 (2002).

2. X. W. Peng, J. L. Ren, L. X. Zhong, F. Peng, and R. C.
Sun, J. Agr. Food Chem., 59, 8208 (2011).

3. N. M. L. Hansen and D. Plackett, Biomacromolecules, 9 , 1493 (2008).

4. X. W. Peng, J. L. Ren, L. X. Zhong, and R. C. Sun, Biomacromolecules, 12, 3321 (2011).

5. O. A. El Seoud, A. Koschella, L. C. Fidale, S. Dorn, and T. Heinze, Biomacromolecules, 8, 2629 (2007).

6. R. P. Swatloski, S. K. Spear, J. D. Holbrey, and R. D. Rogers, J. Am. Chem. Soc., 124, 4974 (2002).

7. Q. Ren, J. Wu, J. Zhang, J. S. He, and M. L. Guo, Acta Polym. Sin., 3, 448 (2003).

8. H. M. Luo, Y. Q. Li, and C. R. Zhou, Polym. Mater. Sci. Eng., 21, 233 (2005).

9. Y. Fukaya, K. Hayashi, M. Wada, and H. Ohno, Green Chem., 10, 44 (2008).

10. F. Lu, B. W. Cheng, J. Song, and Y. Liang, J. Appl. Polym. Sci., 124, 3419 (2012).

11. A. Casas, J. Palomar, M. V. Alonso, M. Oliet, S. Omar, and F. Rodriguez, Ind. Crop. Prod., 37, 155 (2010).

12. H. Xu, W. X. Pan, R. X. Wang, D. J. Zhang, and C. B. Liu, J. Comput. Aid. Mol. Des., 26, 329 (2012).

13. Y. Fukaya, A. Sugimoto, and H. Ohno, Biomacromolecules, 7, 3295 (2006).

14. X. W. Peng, J. L. Ren, and R. C. Sun, Biomacromolecules, 11, 3519 (2011).

15. J. L. Ren, R. C. Sun, C. F. Liu, Z. N. Cao, and W. Luo, Carbohyd. Polym., 70, 406 (2007).

16. X. W. Peng, J. L. Ren, L. X. Zhong, F. Peng, and R. C. Sun, Carbohyd. Polym., 86, 1768 (2011).

17. L. Feng and Z. Chen, J. Mol. Liq., 142, 1 (2008).

18. C. Devallencourt, J. M. Saitern, and D. Capitaine, Polym. Degrad. Stab., 52, 327 (1996).

19. K. Van de Velde and P. Kiekens, J. Appl. Polym. Sci., 83, 2634 (2002).

20. J. F. Dubreuil, K. Bourahla, M. Rahmouni, J. P. Bazureau, and J. Hamelin, Catal. Commun., 3, 185 (2002). 